\title{
Finite-Difference Time-Domain Simulation of Mid- and Far-Infrared Quantum Cascade Lasers
}

\author{
V. TAmošiūnas ${ }^{a, b}, \check{Z}$. $\operatorname{Kancleris}^{a}$, M. Dagys ${ }^{a}$, R. SimnišKis ${ }^{a}$, \\ M. TAmošiunniené $\dot{E}^{a}$, G. VAlušis ${ }^{a}$, G. Strasser ${ }^{b}$ \\ AND K. UNTERRAINER ${ }^{b}$ \\ ${ }^{a}$ Semiconductor Physics Institute, A. Goštauto 11, 01108 Vilnius, Lithuania \\ ${ }^{b}$ Institute of Photonics and Center for Micro- and Nanostructures \\ Vienna University of Technology, 1040 Vienna, Austria
}

\begin{abstract}
We present simulations of mid- and far-infrared quantum cascade lasers operating with/without external magnetic field. Maxwell-Bloch solver based on the finite-difference time-domain method was used in our investigation. Reduction of the far-infrared quantum cascade laser emission intensity is associated with increased optical losses in highly doped layers when magnetic field is changed from $4.2 \mathrm{~T}$ to $6.2 \mathrm{~T}$. A simulated emission spectrum of mid-infrared disc-shaped quantum cascade laser with $60 \mu \mathrm{m}$ radius is consistent with the experimentally observed irregular spacing between quantum cascade laser emission lines.
\end{abstract}

PACS numbers: 72.30.+q, 78.66.--w, 85.30.De

\section{Introduction}

Quantum cascade lasers (QCLs) are coherent sources of mid- and far-infrared radiation based on semiconductor heterostructures. The first operating QCL was demonstrated by Faist et al. in 1994 [1], and the operation of QCL in terahertz range was demonstrated in 2002 [2]. Further development of QCLs is the challenge for several reasons related to intersubband population dynamics and waveguide properties of the laser. Therefore, comprehensive simulations including all laser components (active material, waveguide, etc) are usually required to predict/explain the device performance.

The most common resonators for QCLs are Fabry-Perot cavities with two cleaved facets acting as parallel mirrors reflecting light back from the surface to the active structure. Reflectivity of resonator mirrors should be maximized to reduce the waveguide loss and at the same time the threshold current and volume of 
the active material required for laser operation. One example of lasers exploiting so called "whispering-gallery" modes are micro-disc semiconductor lasers [3]. In such mode light circulates around the inner boundary of the resonator nearly fully reflecting from it with just minimal losses due to evanescent leakage and scattering from the surface roughness.

A finite-difference time-domain (FDTD) method is widely used to simulate the interaction of electromagnetic waves and semiconductor devices and can be efficiently employed to simulate QCLs with above-mentioned waveguide types. It is worth noting that this method permits a relatively easy integration of additional equations describing gain or absorption properties of the simulated material. For example, Ziolkowski et al. suggested [4] a solution scheme for Maxwell-Bloch equations system within the FDTD approach.

\section{Investigation method}

Within the FDTD method [5] centered finite-difference second-order accurate expressions are used to evaluate partial derivatives in space and time:

$$
\begin{aligned}
& \frac{\partial u}{\partial x}(i \Delta x, j \Delta y, k \Delta z, n \Delta t)=\frac{u_{i+1 / 2, j, k}^{n}-u_{i-1 / 2, j, k}^{n}}{\Delta x}+O\left[(\Delta x)^{2}\right], \\
& \frac{\partial u}{\partial t}(i \Delta x, j \Delta y, k \Delta z, n \Delta t)=\frac{u_{i, j, k}^{n+1 / 2}-u_{i, j, k}^{n-1 / 2}}{\Delta t}+O\left[(\Delta t)^{2}\right] .
\end{aligned}
$$

The application of finite-differences to Maxwell equations leads to simple expressions like (only electric field $E_{z}$ in $1 \mathrm{D}$ case is shown):

$$
\left.E_{z}\right|_{i, j} ^{n+1}=\left.E_{z}\right|_{i, j} ^{n}+\frac{\Delta t}{\varepsilon \varepsilon_{0}}\left(\frac{\left.H_{y}\right|_{i+1 / 2, j} ^{n+1 / 2}-\left.H_{y}\right|_{i-1 / 2, j} ^{n+1 / 2}}{\Delta x}-\left.J_{z}\right|_{i, j} ^{n+1 / 2}\right),
$$

here $H_{y}, a J_{z}, a \Delta t, a \Delta x$ are magnetic field and current density components, time and coordinate increments, respectively. Magnetic field and electric field components are computed at points shifted by $1 / 2$ of the space increment. An additional shift by $1 / 2$ of step in a time scale permits a direct computation of the new value of electric field $E_{z}^{n+1}$ from already available values of electric and magnetic field.

Ziolkowski et al. suggested [4] an efficient extension of the FDTD method for two level quantum systems, which permits a simultaneous simulation of Maxwell and Bloch equations within the FDTD cycle:

$$
\left\{\begin{array}{l}
\frac{\partial E_{x}}{\partial t}=-\frac{1}{\varepsilon_{0}} \frac{\partial H_{y}}{\partial z}-\frac{N_{\text {atom }} \gamma}{\varepsilon_{0} T_{2}} \rho_{1}+\frac{N_{\text {atom }} \gamma \omega_{0}}{\varepsilon_{02}} \rho_{2} \\
\frac{\partial \rho_{1}}{\partial t}=-\frac{\rho_{1}}{T_{2}}+\omega_{0} \rho_{2} \\
\frac{\partial \rho_{2}}{\partial t}=-\frac{\rho_{2}}{T_{2}}-\omega_{0} \rho_{1}+2 \frac{\gamma}{\hbar} E_{x} \rho_{3} \\
\frac{\partial \rho_{3}}{\partial t}=-2 \frac{\gamma}{\hbar} E_{x} \rho_{2}-\frac{1}{T_{1}}\left(\rho_{3}-\rho_{30}\right)
\end{array} .\right.
$$

Values of the electric field component $E_{x}$, dispersive and absorptive polariza- 
tions $\rho_{1}, a \rho_{2}$ and the fractional difference of populations $\rho_{3}$ are evaluated using Eqs. $(1,2)$ and updated in a similar to (3) manner using a predictor-corrector algorithm.

Current density term $J$ is usually accounted within FDTD by just properly selecting coefficients in (3) according to Ohm's law for low frequency cases. However, other schemes (like the one based on collision frequency [6]) are also available for high frequency simulations.

Generally, FDTD simulations require vast amounts of computer memory and computation time. Therefore, hybrid techniques like body-of-revolution FDTD (BOR-FDTD) for systems with rotational symmetry were developed [5]. Within BOR-FDTD, Fourier-like expansion into modes is used to account analytically azimuthal variation of electromagnetic field:

$$
\boldsymbol{E}=\sum_{m=0}^{\infty}\left(\boldsymbol{e}_{u} \cos m \phi+\boldsymbol{e}_{v} \sin m \phi\right),
$$

here $\boldsymbol{e}_{u}, a \boldsymbol{e}_{v}, a \boldsymbol{h}_{u}$, and $\boldsymbol{h}_{v}$ are Fourier coefficients dependent on cylindrical coordinates $r, a z$ and time $t$. Such technique dramatically reduces computer requirements, but also prohibits non-linearity of update equations. Therefore, only small amplitude/large inversion ( $\rho_{3} \approx$ const) cases can be simulated within BOR-FDTD. A similar approximation can be applied also to Fabry-Perot resonator modes.

\section{Simulations of disc-shaped QCLs}

Production of ring- and disc-shaped QCLs is one of the most attractive options, when exceptional performance and low threshold currents are required. The experimental study [7] confirms that microcylinder QCLs compare favorably to both Fabry-Perot and distributed feedback cavities that were processed from the same material. A low threshold operation is expected for "whispering-gallery" modes, but the irregularity of emission spectrum [7] and influence of changing inner radius in case of ring resonator [8] suggest that a more detailed study is needed to fully understand the observed phenomena.

Figure 1 summarizes BOR-FDTD simulation results for disc-shaped mid-IR QCL from Ref. [7]. As expected, a "whispering-gallery" mode solution is obtained for mode numbers $m=86 \ldots 90$. However, higher-order solutions with similar amplitudes are obtained for $m<85$. In addition, additional emission lines of these higher-order modes (Fig. 1 right) can distort a regular spacing between "whispering-gallery" modes (Fig. 1 middle) as observed.

In addition, left part of Fig. 1 can also explain why emission spectra of the ring-shaped QCLs are affected by changing radius of the inner etched a area [8]. As one can clearly see, modes of QCL can propagate quite far from the outer boundary. Therefore, a number of possible modes is reduced when the central part of the disc is removed by etching. Such restrictions lead to the appearance of other modes in the emission spectrum as observed. 

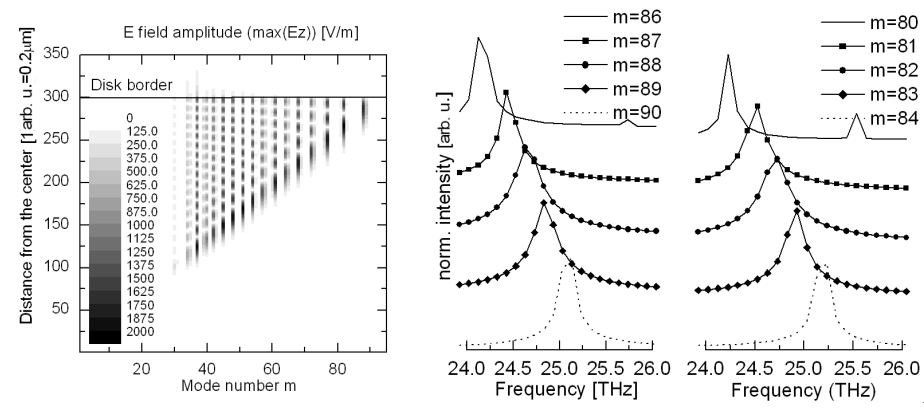

Fig. 1. Computed amplitudes (left) and spectra (right) of various mid-IR microcylinder QCL modes. Parameters of the actual QCL were used as a basis [7]. Amplitudes were normalized to the same values at the right side of the figure for easier comparison of frequencies.

\section{4. $\mathrm{THz}$ QCLs in magnetic field}

Simulation results for fixed gain medium parameters and two possible waveguide designs of the THz QCL are presented in Fig. 2. These lasers rely on a careful selection of doping and thickness for bottom $n^{+}$layer, since the mode confinement and losses in case of this QCL design are strongly dependent on the dielectric constant of the $n^{+}$layer. Therefore, FDTD simulations were employed to compare two possible designs. It is clearly seen that, while slightly higher losses are expected for $500 \mathrm{~nm}$ thick $4 \times 10^{18} \mathrm{~cm}^{-3}$ doped $n^{+}$layer at $0 \mathrm{~T}$ when compared with the original design suggested in [2], the broader operation range is expected in a magnetic field.

A recent experimental study of $\mathrm{THz}$ QCLs with such waveguide in magnetic field [9] revealed several interesting effects, like emission line shifts and modulation of the emission intensity. A clear emission intensity maximum was observed at
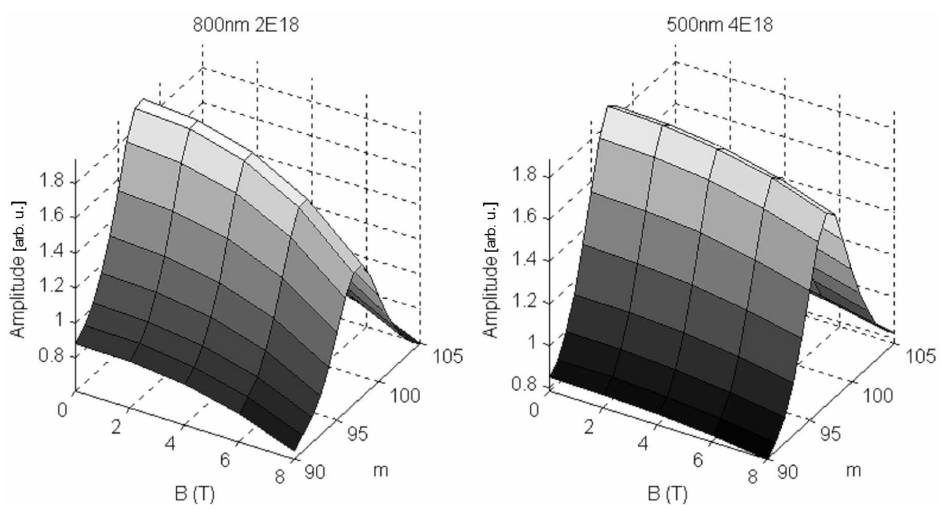

Fig. 2. Achieved amplitude versus mode number $m$ and magnetic field $B(T)$ for two waveguide designs. 
4.2 T, which was followed by the reduced intensity peak at $6.2 \mathrm{~T}$. Such reduction can clearly be attributed to an increased loss in the waveguide, while the active structure of the material was expected to perform better above $5 \mathrm{~T}$.

\section{Acknowledgments}

Authors would like to acknowledge the support by the Austrian Science Fund (START, ADLIS SFB), European Union Framework VI programme (TERANOVA, ANSWER), EU Center of Excellence in Processing, Research and Application of Advanced Materials (PRAMA) and Lithuanian State Science and Studies Foundation ("Advanced Millimeter Wave Electronics").

\section{References}

[1] J. Faist, F. Capasso, D.L. Sivco, C. Sirtori, A.L. Hutchinson, A.Y. Cho, Science 264, 553 (1994).

[2] R. Khler, A. Tredicucci, F. Beltram, H.E. Beere, E.H. Linfield, A.G. Davies, D.A. Ritchie, R.C. Iotti, F. Rossi, Nature 417, 156 (2002).

[3] Y. Yamamoto, R.E. Slusher, Physics Today 46, 66 (1993).

[4] R.W. Ziolkowski, J.M. Arnold, D.M. Gogny, Phys. Rev. A 52, 3082 (1995).

[5] A. Taflove, Computational Electrodynamics: The Finite-Difference Time-Domain Method, Artech House, Norwood (MA) (USA) 1995 (and references within).

[6] J.L. Young, Radio Sci. 29, 1513 (1994).

[7] S. Anders, W. Schrenk, E. Gornik, G. Strasser, Appl. Phys. Lett. 80, 4094 (2002).

[8] S. Anders, V. Tamosiunas, W. Schrenk, G. Strasser, Phys. Rev. B 69, 073309 (2004).

[9] V. Tamosiunas, R. Zobl, J. Ulrich, K. Unterrainer, R. Colombelli, C. Gmachl, K. West, L. Pfeiffer, F. Capasso, Appl. Phys. Lett. 83, 3873 (2003). 